\title{
Chemical and physical properties of gall stones in South Australia: implications for dissolution treatment
}

\author{
M J WHITING, * B M BRADLEY, and J McK WATTS \\ From the Department of Surgery, Flinders Medical Centre, Flinders University of South Australia, South \\ Australia
}

SUMMARY Of 406 consecutive patients with gall-bladder stones, 387 were treated by cholecystectomy and 19 were treated with chenodeoxycholic acid. The gall stones found in 356 of the 387 patients at operation were analysed chemically for cholesterol and calcium, physically for number, size, and mass, and radiologically before cholecystectomy for stone lucency. Gall stones rich in cholesterol ( $>80 \%$ by weight) were present in $75 \%$ of patients, while pigment stones ( $<25 \%$ cholesterol) were found in $12 \%$ of patients. Out of the 406 gall-stone patients, $77(19 \%)$ had functioning gall bladders which contained radiolucent stones $1.5 \mathrm{~cm}$ or less in diameter, thereby fulfilling the criteria which are used by most clinicians in selecting patients for dissolution therapy of cholesterol gall stones. The stones of 61 of these patients were available for chemical analysis and $52(85 \%)$ were rich in cholesterol. Using the more stringent criterion for stone size of $1 \mathrm{~cm}$ or less in diameter, only $11 \%$ of patients had stones suitable for dissolution treatment. Taking into account other factors such as lack of compliance with treatment, obesity, and calcium salts in gall stones, it appears that no more than $10 \%$ of gall-stone patients presenting to a general hospital could be successfully treated with chenodeoxycholic acid.

The gall stones of Western man are usually composed predominantly of cholesterol with small amounts of other components, such as calcium salts and bile pigment. ${ }^{12}$ Some gall stones, however, have a low cholesterol content (less than $25 \%$ by weight), and are termed pigment stones. ${ }^{3}$ The incidence of pigment stones has been generally accepted as around $10 \%$ of the gall-stone population, ${ }^{3}$ but recent studies indicate that approximately $30 \%$ of gall-stone patients in the USA have pigment stones. ${ }^{4}$

With the advent of chenodeoxycholic acid therapy for the dissolution of cholesterol gall stones, ${ }^{5-7}$ it is important to clarify the incidence of cholesterol $v s$ pigment gall-stone disease as a starting point to determine the number of patients who could potentially benefit from this new treatment. In this study we analysed gall-bladder stones collected at cholecystectomy from a large series of patients for cholesterol and calcium. As well as gaining information on the chemical properties of gall stones

\footnotetext{
* Address for correspondence: M J Whiting, PhD, Department of Surgery, Flinders Medical Centre, Bedford Park, South Australia 5042.

Received for publication 13 April 1982
}

in South Australia, we recorded some physical properties such as stone size and total stone mass, and stone radiolucency or radio-opacity, after radiological examination before cholecystectomy. All these factors are known to be of great importance in determining the outcome of dissolution treatment. From this information, an estimate has been made of the percentage of patients presenting to a general hospital in South Australia who could potentially be offered dissolution rather than surgical treatment for their gall-stone disease.

\section{Methods}

ANALYSIS OF GALL STONES

Gall-bladder stones were present in 387 cholecystectomies performed at Flinders Medical Centre over a period of 30 months. The stones were sent to the laboratory from 356 patients (92\%), where they were washed with water, dried, weighed, and the diameter of the largest stone measured. Total stone cholesterol and calcium (\% by weight) was determined as previously described ${ }^{8}$ after grinding several stones, or half a stone when only one was present, to a fine powder with a mortar and pestle. 
Stone radiolucency and gall-bladder function were determined by plain radiograph and oral cholecystography before cholecystectomy.

\section{DATA ANALYSIS}

Patient data collected included age at cholecystectomy, sex, height, and weight. Gall stone and patient data were stored in a DEC-10 computer (Digital Equipment Corp., Mass., USA) and analysed using an SPSS Statistics package. The statistical test used was the non-parametric MannWhitney U test.

\section{Results}

CHEMICAL CHARACTERISTICS OF GALL STONES Gall stones were divided into three groups according to their cholesterol content. These three groups were (1) high cholesterol (greater than $80 \%$ by weight); (2) medium cholesterol (between 25 and $80 \%$ ); and (3) low cholesterol or pigment (less than $25 \%)$. As seen in Table 1, by this classification, high cholesterol stones were present in $75 \%$ of all patients, while stones of medium and low cholesterol content were found in 13 and $12 \%$ of patients respectively. The low cholesterol stones had the typical appearance of pigment stones, ${ }^{3}$ and were usually small and multiple, irregular in shape, and were black to brown in colour. In four patients, two morphologically distinct types of stones were present in the gall bladder. Chemical analysis of these stones confirmed that they were markedly different in both cholesterol and calcium content. Two patients had high cholesterol plus pigment stones; one patient had high and medium cholesterol stones; and one patient had medium cholesterol and pigment stones. The stones of these four patients were excluded from further analysis in this study.

The calcium content was examined in each of the three groups, and was significantly higher $(p<0.001)$ in the medium and low cholesterol groups than in

Table 1 Cholesterol and calcium content of gall stones collected from 352 patients

\begin{tabular}{|c|c|c|c|}
\hline & \multicolumn{3}{|c|}{ Stone cholesterol content } \\
\hline & Low & Medium & High \\
\hline Patients (no.) & $41(12 \%)$ & $45(13 \%)$ & $266(75 \%)$ \\
\hline \multicolumn{4}{|l|}{ Cholesterol content (\%) } \\
\hline Median & 4 & 68 & 92 \\
\hline Range & $0-25$ & $26-79$ & $80-100$ \\
\hline \multicolumn{4}{|l|}{ Calcium content (\%) } \\
\hline Median & $3 \cdot 0$ & $6 \cdot 0$ & $0 \cdot 3$ \\
\hline Range & $0-35.4$ & $0 \cdot 2-21 \cdot 6$ & $0-6 \cdot 1$ \\
\hline
\end{tabular}

the high cholesterol group (Table 1). In each group, there was neither a significant difference in the calcium content of stones between males and females, nor was there any relationship between the age of the patients at cholecystectomy and the calcium content of their stones.

\section{PHYSICAL CHARACTERISTICS OF GALL STONES}

The diameter of the largest stone present in the gall bladders of the 352 gall-stone patients was measured in the laboratory, and is shown in Fig. 1. Over one-third of the patients $(39 \%)$ had at least one stone greater than $1.5 \mathrm{~cm}$ in diameter, and large stones were found in $44 \%$ of the patients in the high cholesterol group. In contrast, small stones $1.5 \mathrm{~cm}$ or less in diameter were found in $95 \%$ of patients with low cholesterol stones.

Most patients $(82 \%)$ had multiple stones as opposed to a solitary stone, and there was no significant difference in the proportion of patients with multiple stones in the groups of different stone cholesterol content. In the high cholesterol group, the total mass of the stones and the diameter of the largest stone increased with the age of the patient (Table 2). These increases were highly significant $(p<0.001)$ when comparing patients less than 40 with patients greater than 60 years of age.

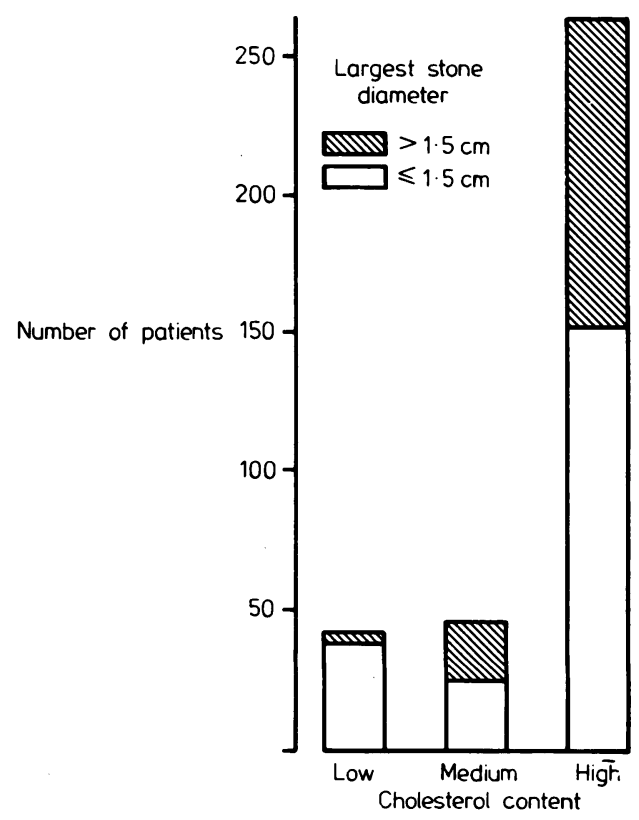

Fig. 1 Diameter of the largest stone present in the gall bladders of 352 patients with stones of different cholesterol content. 
Table 2 Total gall-bladder stone mass and diameter of largest stone according to age in 266 patients with stones of high cholesterol content

\begin{tabular}{|c|c|c|c|c|c|}
\hline \multirow{2}{*}{$\begin{array}{l}\text { Age group } \\
(y r)\end{array}$} & \multirow{2}{*}{$\begin{array}{l}\text { Patients } \\
\text { (no.) }\end{array}$} & \multicolumn{2}{|c|}{$\begin{array}{l}\text { Total mass } \\
\text { of stones }(\mathrm{g})\end{array}$} & \multicolumn{2}{|c|}{$\begin{array}{l}\text { Diameter of } \\
\text { largest stone }(\mathrm{cm})\end{array}$} \\
\hline & & Median & Range & Median & Range \\
\hline$<40$ & 89 & $2 \cdot 0$ & $0 \cdot 1-32 \cdot 5$ & $1 \cdot 3$ & $0 \cdot 3-3 \cdot 5$ \\
\hline $40-60$ & 83 & $3 \cdot 8$ & $0 \cdot 1-34 \cdot 3$ & $1 \cdot 5$ & $0 \cdot 2-3 \cdot 9$ \\
\hline$>60$ & 94 & $5 \cdot 0$ & $0 \cdot 2-47 \cdot 0$ & 1.6 & $0 \cdot 3-7 \cdot 0$ \\
\hline
\end{tabular}

Reports from gall-bladder radiology were available in 331 of the 352 patients, and $76 \%$ of all patients had radiolucent stones. When considered according to their cholesterol content, $84 \%$ of the high cholesterol stones were radiolucent, as were $36 \%$ of the medium cholesterol stones and $59 \%$ of the lower cholesterol (pigment) stones (Fig. 2). These results were in keeping with the calcium content of the stones. Thus, $86 \%$ of all radiolucent stones were high in cholesterol, which agrees with previous studies by Bell et al, ${ }^{9}$ who found that in 20 out of 25 patients with radiolucent stones, the stones were cholesterol in type.

EPIDEMIOLOGICAL ASPECTS RELATING TO STONE TYPE

Although the age range at cholecystectomy was

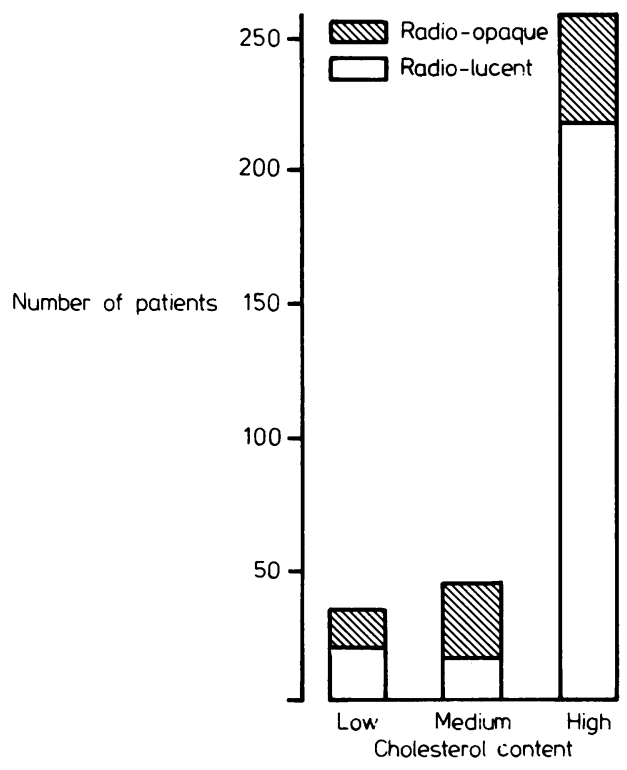

Fig. 2 Radiolucency of gall-bladder stones of different cholesterol content. similar for patients with gall stones of different cholesterol content, the median age for patients with low cholesterol stones was significantly greater $(p<0 \cdot 001)$ than the high cholesterol group (Table 3). The sex ratio was also different. Low cholesterol (pigment) stones were almost equally distributed among males and females (Table 3), while high cholesterol stones were more frequently found among females, especially in the age range from 20 to 40 years, where the sex ratio approached $4: 1$ (Fig. $3)$.

The body mass index (weight/height ${ }^{2}$ ) as a measure of obesity was not significantly greater in the high cholesterol stone group (median 25.1, range 18.0-48.9) than in the low cholesterol stone group (median $24 \cdot 5$, range $17 \cdot 3-31 \cdot 2$ ).

\section{Discussion}

The chemical composition of gall-bladder stones in South Australia is such that stones rich in cholesterol are very common, with $75 \%$ of all patients having stones containing over $80 \%$ cholesterol by weight. On the other hand, pigment stones (defined as containing less than 25\% cholesterol) are relatively rare. The incidence of $12 \%$ is considerably lower than in the USA where it has been reported that $27 \%$ of all stones are pigment in type. ${ }^{4}$

Some other features of pigment stone epidemiology which contrast markedly with features for cholesterol stones have been confirmed in this study. They are the appearance of symptoms from pigment stones later in life than for cholesterol stones (as judged by presentation for cholecystectomy) and the almost equal distribution among men and women. For cholesterol stones, the overall female to male sex ratio was $2 \cdot 1$, and in the less than 40 years age group, the ratio was even higher at 3.5. This is consistent with suggestions that female sex hormones, and in particular oral contraceptives, are predisposing factors for cholesterol gall-stone formation. ${ }^{10}$ Although obesity is also thought to be a

Table 3 Age at cholecystectomy and sex distribution of 352 patients with gall stones of different cholesterol content

\begin{tabular}{|c|c|c|c|}
\hline & \multicolumn{3}{|c|}{ Stone cholesterol content } \\
\hline & Low & Medium & High \\
\hline Patients (no.) & 41 & 45 & 266 \\
\hline \multicolumn{4}{|c|}{ Age (yr) at cholecystectomy } \\
\hline Median & 62 & 52 & 51 \\
\hline Range & $21-80$ & $25-86$ & $18-85$ \\
\hline \multicolumn{4}{|l|}{ Sex ratio } \\
\hline Females/males & $1 \cdot 2$ & $2 \cdot 3$ & $2 \cdot 1$ \\
\hline
\end{tabular}




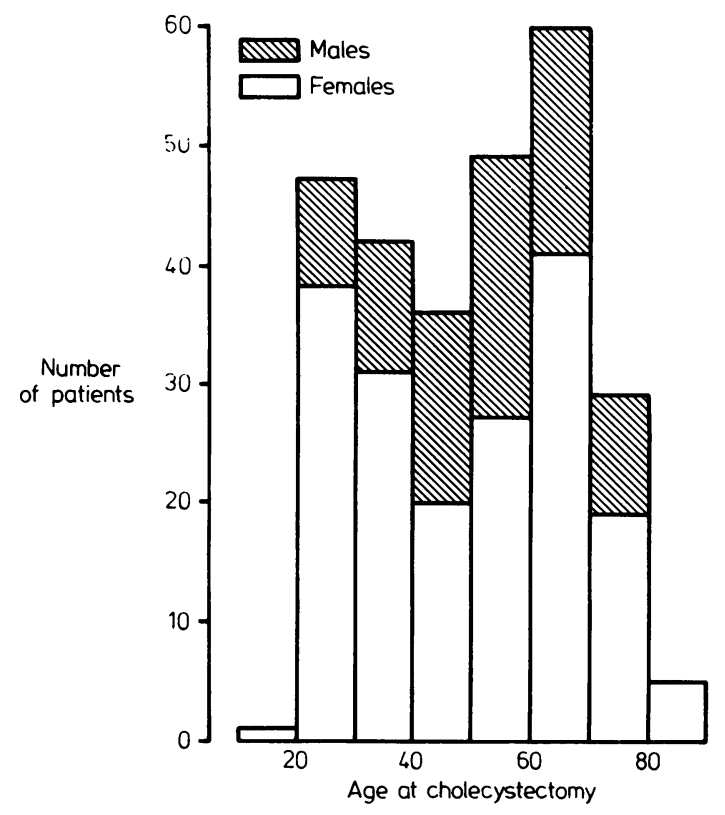

Fig. 3 Age at cholecystectomy and sex distribution of 266 patients with gall stones containing $80 \%$ or more cholesterol.

risk factor for cholesterol gall-stone disease, our results show that patients with cholesterol gall stones are not significantly more obese than patients with pigment stones.

The physical and chemical properties of gall stones described in this study have important implications when considering the future of dissolution treatment with chenodeoxycholic or ursodeoxycholic acid for cholesterol gall-stone disease. Several clinical trials with chenodeoxycholic acid have now determined that only patients without acute symptoms and with small radiolucent stones $(1.5 \mathrm{~cm}$ or less in diameter) in a functioning gall bladder should be considered for this form of treatment. ${ }^{5-7}$ Of the 387 consecutive gall-stone patients in this study who were treated surgically, 63 $(16 \%)$ fulfilled these criteria. Furthermore, if these 63 patients had not undergone cholecystectomy but had been treated with chenodeoxycholic acid, it is likely that only around 50 to $70 \%$ of them would have dissolved their stones. This is because the stones of 61 of these 63 patients were chemically analysed, and only 52 were found to be high in cholesterol content. Other factors such as lack of patient compliance with treatment, layers of calcium salts on the surface of radiolucent stones ${ }^{8}$ or obesity ${ }^{11}$ can also prevent dissolution.

While conducting this survey of the properties of gall-bladder stones collected at cholecystectomy, 19 additional gall-stone patients with radiolucent stones in a functioning gall bladder were treated with chenodeoxycholic acid instead of undergoing surgery. Only 14 of these patients, however, had stones $1.5 \mathrm{~cm}$ or less in diameter. The percentage of patients who presented to our hospital over the 30 month period of this study and were suitable for dissolution therapy should therefore be corrected to $(63+14) /(387+19)$ or $19 \%$. If the criterion of stone size is made more stringent so that only patients with stones $1 \mathrm{~cm}$ or less in diameter are acceptable, then $11 \%$ of the patients in this study would have been suitable for dissolution treatment. Although large stones can occasionally be dissolved, the efficacy of gall-stone dissolution is related to stone size,$^{5-7}$ and stones larger than $1 \mathrm{~cm}$ in diameter may take two years or more to dissolve. Such a long and expensive course of treatment will be unacceptable to many patients.

We conclude that the overall impact of chenodeoxycholic acid on the treatment of gall-stone disease in Australia will be small. Less than $19 \%$ of gall-stone patients presenting to hospital have functioning gall bladders which contain stones that exhibit the physical and chemical characteristics required for successful dissolution treatment with this bile acid. When other patient-related factors are taken into account, we estimate that, in practice, no more than $10 \%$ of gall-stone patients presenting to our hospital could have their gall stones completely dissolved by chenodeoxycholic acid treatment.

We would like to thank Mr V Jarvinen for technical assistance with the gall-stone analyses. The chenodeoxycholic acid used during the period of this study was kindly supplied by Weddel Pharmaceuticals, London.

\section{References}

1 Shaffer EA. Gallstones: current concepts of pathogenesis and medical dissolution. Can J Surg 1980; 23: 517-32.

2 Sutor DJ, Wooley SE. A statistical survey of the composition of gallstones in eight countries. Gut 1971; 12: $55-64$.

3 Soloway RD, Trotman BW, Ostrow JD. Pigment gallstones. Gastroenterology 1977; 72: 167-82.

4 Trotman BW, Soloway RD. Pigment vs cholesterol cholelithiasis: clinical and epidemiological aspects. $\mathrm{Am}$ J Dig Dis 1975; 20: 735-40.

5 Dowling RH. Chenodeoxycholic acid therapy of gallstones. Clin Gastroenterol 1977; 6: 141-63.

6 Toouli J, Jablonski P, Watts JMcK. Treatment of 
gallstones by chenodeoxycholic acid. Med J Aust 1980; 1: 478-9.

7 Schoenfield LJ, Lachin JM. The Steering Committee and the National Cooperative Gallstone Study Group. Chenodiol (chenodeoxycholic acid) for dissolution of gallstones. The National Cooperative Gallstone Study. Ann Intern Med 1981; 95: 257-82.

8 Whiting MJ, Jarvinen V, Watts JMcK. Chemical composition of gallstones resistant to dissolution therapy with chenodeoxycholic acid. Gut 1980; 21: 1077-81.
9 Bell GD, Dowling RH, Whitney B, Sutor DJ. The value of radiology in predicting gallstone type when selecting patients for medical treatment. Gut 1975; 16: 359-64.

10 Bennion LJ, Grundy SM. Risk factors for the development of cholelithiasis in man. N Engl J Med 1978; 299: 1221-7.

11 Iser JH, Maton PN, Murphy GM, Dowling RH. Resistance to chenodeoxycholic acid treatment in obese patients with gallstones. $\mathrm{Br} \operatorname{Med} J$ 1978; 1: 1509-12. 